\title{
Cerebrospinal Fluid Alzheimer Markers in Depressed Elderly Subjects with and without Alzheimer's Disease
}

\author{
Milica Gregoric Kramberger ${ }^{a, c}, \mathrm{e}$ Vesna Jelic ${ }^{b, c}$ Ingemar Kåreholt ${ }^{d}$ \\ Daniela Enache ${ }^{a, f}$ Maria Eriksdotter Jönhagen ${ }^{b, c}$ Bengt Winblad ${ }^{a, c}$ \\ Dag Aarsland ${ }^{\mathrm{a}, \mathrm{c}, \mathrm{g}}$
}

\begin{abstract}
${ }^{a}$ Alzheimer Disease Research Center and b Division of Clinical Geriatrics, Department of Neurobiology, Care Sciences and Society, Karolinska Institutet, ' Department of Geriatric Medicine, Karolinska University Hospital, and ${ }^{d}$ Aging Research Center, Karolinska Institutet/Stockholm University, Stockholm, Sweden; ${ }^{e}$ Department of Neurology, University Medical Center Ljubljana, Ljubljana, Slovenia; ${ }^{f}$ Clinical Hospital of Psychiatry Prof. dr. Alexandru Obregia, Bucharest, Romania; ${ }^{9}$ Centre for Age-Related Medicine, Stavanger University Hospital, Stavanger, Norway
\end{abstract}

\section{Key Words}

Depression • Elderly $\cdot$ Alzheimer's disease $\cdot$ Cerebrospinal fluid $\cdot \beta$-Amyloid 1-24 $\cdot$ Total tau Phosphorylated tau

\begin{abstract}
Background: The aim of this study was to explore the relationship between cerebrospinal fluid Alzheimer's disease (AD) markers and depression in elderly people. Method: We included subjects with $A D$ as well as persons with subjective cognitive impairment and normal cognition. Depression was assessed with the Cornell Scale for Depression in Dementia, and a cut-off score of $>6$ was used to define depression. Cerebrospinal fluid was analyzed using commercially available assays for $\beta$-amyloid 1-42, total tau, and phosphorylated tau 181. Results: A total of 183 participants ( $66.7 \%$ female) were included ( 92 with AD and 91 with subjective cognitive impairment), with a mean age $( \pm S D)$ of $67.6 \pm 7.4$ years, a Mini-Mental State Examination score of $26.0 \pm 4.0$, and a median Cornell Scale for Depression in Dementia score of 5 (range 0-19). Depression scores were not associated with higher phosphorylated tau 181 and total tau or reduced $\beta$-amyloid 1-42 in AD or non-demented subjects. Conclusions: These results suggest that $A D$ pathology does not contribute to depression, indicating that other factors may be more important. Further studies of the aetiology of depression in elderly people with and without AD are warranted.

Copyright $\odot 2012$ S. Karger AG, Basel
\end{abstract}




\section{Introduction}

Depression is one of the most common health problems in the elderly, and non-major depression syndromes such as minor depression occur in $4-13 \%$, dysthymia in $2 \%$, and clinically significant symptoms of depression in $8-16 \%$ of patients, with major implications for quality of life, physical health, independent functioning, and costs [1].

There is a complex relationship between late-onset depression and cognitive impairment. Executive dysfunction is common in depressed elderly subjects, and depression is more prevalent among patients with Alzheimer's disease (AD) and other dementias than in non-demented elderly subjects; furthermore, there is emerging evidence that mid-life depression is a risk factor for AD decades later [2]. Finally, in many studies, depression has been found to be a prodromal syndrome of early $\operatorname{AD}[2,3]$.

The aetiology of late-life depression is complex and only partially understood. Psychosocial, genetic as well as brain changes probably contribute, and potential mechanisms include toxic stress, with the activation of the hypothalamic-pituitary-adrenal axis, hypercortisolaemia, inflammation, and hippocampus atrophy, monoaminergic changes, and cerebrovascular disease [4].

There is evidence that $\mathrm{AD}$ pathology is a contributing factor to the development of depression in elderly subjects with and without $\mathrm{AD}$, possibly associated with decreased serotonin, which is likely to be related to depression in $\mathrm{AD}$ [5] since serotonin influences the shifting in the processing of amyloid precursor protein towards amyloidogenic pathways $[6$, 7]. MRI studies have reported more hippocampal atrophy in elderly patients with depression [8]. Moreover, clinicopathological studies have reported more AD-type changes in AD patients with a previous history of depression compared to those without a history of depression [9]. The same authors also reported increased tangle pathology in $\mathrm{AD}$ patients with comorbid depression compared to those without depression [10]. Recent studies using amyloid PET imaging have, to some extent, been consistent with these autopsy findings $[11,12]$. However, autopsy studies of late-life depression without dementia have failed to find significantly more AD pathology in depressed compared to non-depressed elderly persons [13]. Thus, the association between depression and $\mathrm{AD}$ pathology is not clear.

In cerebrospinal fluid (CSF), a characteristic pattern of metabolites of amyloid plaques [low $\beta$-amyloid 1-42 (A $\beta 42)$ ] and tau [increased total tau (t-tau) and phosphorylated tau 181 (p-tau)] has been consistently shown in patients with AD [14]. However, the few studies that have explored whether AD-type changes in CSF are associated with depression have not found associations with more severe depression in mild AD [15] or elderly non-demented women [16].

Taken together, there is inconsistent evidence regarding the role of AD-type pathological changes in depression. Understanding the aetiology of depression in the elderly is important for diagnostic purposes as well as background information for the development of novel pharmacological strategies. We administered the Cornell Scale for Depression in Dementia (CSDD) [17] to 183 elderly subjects who had been referred to a memory clinic and diagnosed as having $\mathrm{AD}$ or subjective cognitive impairment [SCI; i.e. normal cognitive functioning without a diagnosis of dementia or mild cognitive impairment (MCI)], and measured CSF levels of $A \beta 42$, $t$-tau, and $p$-tau. The aim of this study was to test the hypothesis that AD-type changes, i.e. reduced $A \beta 42$ and increased t-tau and $p$-tau, are associated with depression in elderly subjects with and without AD. 


\section{Methods}

\section{Subjects}

The base population for the study consisted of 1,154 referrals to the Memory Clinic at Karolinska University Hospital in Huddinge, Sweden, between 2007 and 2009. Inclusion criteria for the current project were: (a) age 60 years or older, (b) CSDD administered, (c) lumbar puncture, and (d) diagnosis of $\mathrm{AD}$ or normal cognition (i.e. SCI). Excluded were patients with: (a) physical disease significantly affecting cognitive performance, (b) life-threatening disease with expected reduced survival time, (c) MCI, and (d) dementia due to diseases other than $\mathrm{AD}$.

All subjects signed informed consent prior to lumbar puncture, and the study was approved by the Ethical Committee at Karolinska University Hospital in Huddinge (43/03).

\section{Assessment}

Participants underwent a medical examination and assessment of depression using the combined patient- and informant-based CSDD, which has excellent psychometric properties in both demented and non-demented people $[17,18]$. For this study, a cut-off score of $>6$ was used to define depressed and non-depressed subjects, based on a validation study performed in Denmark [19]. The CSDD was completed by a licensed geriatrician or psychiatrist, or a research nurse.

For the dementia diagnosis, a standardized informant-based scale of the patients' history of cognitive decline (Informant Questionnaire on Cognitive Decline in the Elderly) [20] was administered, and dementia was staged using Global Deterioration Scales after a clinical medical examination including cognitive screening with the Mini-Mental State Examination (MMSE) [21]. To aid in the diagnostic process, the majority of subjects completed a neuropsychological examination: tests of verbal abilities (similarities and information), visuospatial abilities (block design and Rey-Osterrieth copying), working memory (digit span and Corsi span), episodic memory (learning and retention after $30 \mathrm{~min}$, Rey Auditory Learning Test), and executive functioning (Digit Symbol and Trail Making Test). Patients were diagnosed as having AD using ICD 10 criteria after a consensus meeting with clinicians and neuropsychologists, taking into account all available information.

In addition to the clinical assessment, brain imaging MRI or CT according to a dementia protocol, routine blood tests, and CSF analyses were performed; furthermore, EEG was performed in a large subgroup.

\section{CSF Measurement}

CSF was obtained by lumbar puncture between the L3/L4 or L4/L5 intervertebral space using a 25 -gauge needle and collected in 12-ml polypropylene tubes. Within $2 \mathrm{~h}$, the CSF samples were centrifuged. A small amount of CSF was used for routine analysis, including total cells (leucocytes and erythrocytes), total protein, and glucose. CSF was aliquoted in polypropylene tubes of 0.5 or $1 \mathrm{ml}$ and stored at $-80^{\circ} \mathrm{C}$ until further analysis. CSF A $\beta 42, \mathrm{t}-$ tau, and p-tau were measured by commercially available sandwich ELISAs (Innogenetics, Ghent, Belgium).

\section{Statistics}

We divided the subjects into four groups based on the presence or absence of depression in $\mathrm{AD}$ and SCI. Group comparisons were made using parametric or non-parametric tests as appropriate. For the descriptive statistics presented in table 1 a $\chi^{2}$ test and a pre-planned post hoc analysis have been used. The CSF values were compared using simple logistic regression comparing depressed and non-depressed subjects with and without AD in two separate anal- 
Table 1. Characteristics of the study sample

\begin{tabular}{|c|c|c|c|c|c|c|c|}
\hline & \multirow{2}{*}{$\begin{array}{l}\text { All } \\
(\mathrm{n}=183)\end{array}$} & \multicolumn{3}{|l|}{ SCI } & \multicolumn{3}{|l|}{$\mathrm{AD}$} \\
\hline & & $\begin{array}{l}\text { not } \\
\text { depressed } \\
(\mathrm{n}=51)\end{array}$ & $\begin{array}{l}\text { depressed } \\
(\mathrm{n}=41)\end{array}$ & $\mathrm{p}$ & $\begin{array}{l}\text { not } \\
\text { depressed } \\
(\mathrm{n}=60)\end{array}$ & $\begin{array}{l}\text { depressed } \\
(\mathrm{n}=31)\end{array}$ & $\mathrm{p}$ \\
\hline Age, years & $67.6 \pm 7.4$ & $65.0 \pm 5.6$ & $62.7 \pm 3.0$ & 0.02 & $72.0 \pm 8.2$ & $70.1 \pm 6.6$ & 0.26 \\
\hline Females & $122(66.7)$ & $32(62.7)$ & $32(78.0)$ & 0.11 & $38(63.3)$ & $20(64.5)$ & 0.9 \\
\hline Education, years & $11.7 \pm 3.7$ & 13.4 & 12.4 & 0.19 & 11.0 & 11.2 & 0.8 \\
\hline MMSE score & $26.0 \pm 4.0$ & $28.9 \pm 1.4$ & $28.7 \pm 1.5$ & 0.5 & $23.1 \pm 3.6$ & $23.2 \pm 3.9$ & 0.9 \\
\hline $\mathrm{AD}$ duration, years & $3.2 \pm 2.3$ & 3.0 & 3.2 & 0.6 & 3.0 & 3.4 & 0.5 \\
\hline CSDD score & $5.0(0-19)$ & $3(0-6)$ & $11(7-19)$ & $<0.0005$ & $2(0-6)$ & $9(7-14)$ & $<0.0005$ \\
\hline Antidepressant use & $61(33.3)$ & $10(19.6)$ & $12(29.3)$ & 0.28 & $26(43.3)$ & $13(41-9)$ & 0.9 \\
\hline
\end{tabular}

Numbers represent mean \pm SD, $n(\%)$, or median (range).

Between-group comparisons were made using Mann-Whitney or $\chi^{2}$ tests as appropriate.

Table 2. CSF markers and depression in subjects with and without AD

\begin{tabular}{llllllll}
\hline & SCI & & & & AD & & \\
\cline { 2 - 3 } & $\begin{array}{l}\text { not depressed } \\
(\mathrm{n}=51)\end{array}$ & $\begin{array}{l}\text { depressed } \\
(\mathrm{n}=41)\end{array}$ & $\mathrm{p}^{*}$ & & $\begin{array}{l}\text { not depressed } \\
(\mathrm{n}=60)\end{array}$ & $\begin{array}{l}\text { depressed } \\
(\mathrm{n}=30)\end{array}$ & $\mathrm{p}^{*}$ \\
\hline $\mathrm{p}$-tau, pg/l & $57.3(31)$ & $50.1(23)$ & 0.045 & & $85.5(42)$ & $79.5(31)$ & 0.256 \\
t-tau, pg/l & $302(157)$ & $237(145)$ & 0.023 & $560.5(496)$ & $439(240)$ & 0.027 \\
$\mathrm{~A} \beta 42, \mathrm{pg} / 1$ & $883(372)$ & $896(298)$ & 0.897 & & $504(160)$ & $429(154)$ & 0.157 \\
\hline
\end{tabular}

Numbers represent median (interquartile range).

* p values are based on logistic regressions with depressed/not depressed as outcome.

yses. In addition, Spearman's correlation analyses were performed between the CSDD score and each of the three CSF measures in the total group and in the AD and SCI groups separately. Ordered logistic regression was used to analyze the association between CSF measures and depression scores using the entire CSDD scale. Two models were used, adjusting for age and sex (Model 1) and age, sex, and MMSE score (Model 2).

\section{Results}

The sample included 183 patients. The characteristics of the four groups are shown in table 1. Age, MMSE score, and CSDD score, but not gender, differed significantly between the groups. The pre-planned post hoc analyses showed that in AD and SCI, the only significant difference was in the non-demented group, with a lower age in the depressed compared to the non-depressed subjects (table 1).

\section{CSF Analyses}

The median values of the three CSF measures in the four groups are shown in table 2 and figures 1-3. As expected, in the AD groups, $t$-tau and $\mathrm{p}$-tau were higher and $\mathrm{A} \beta 42$ low- 

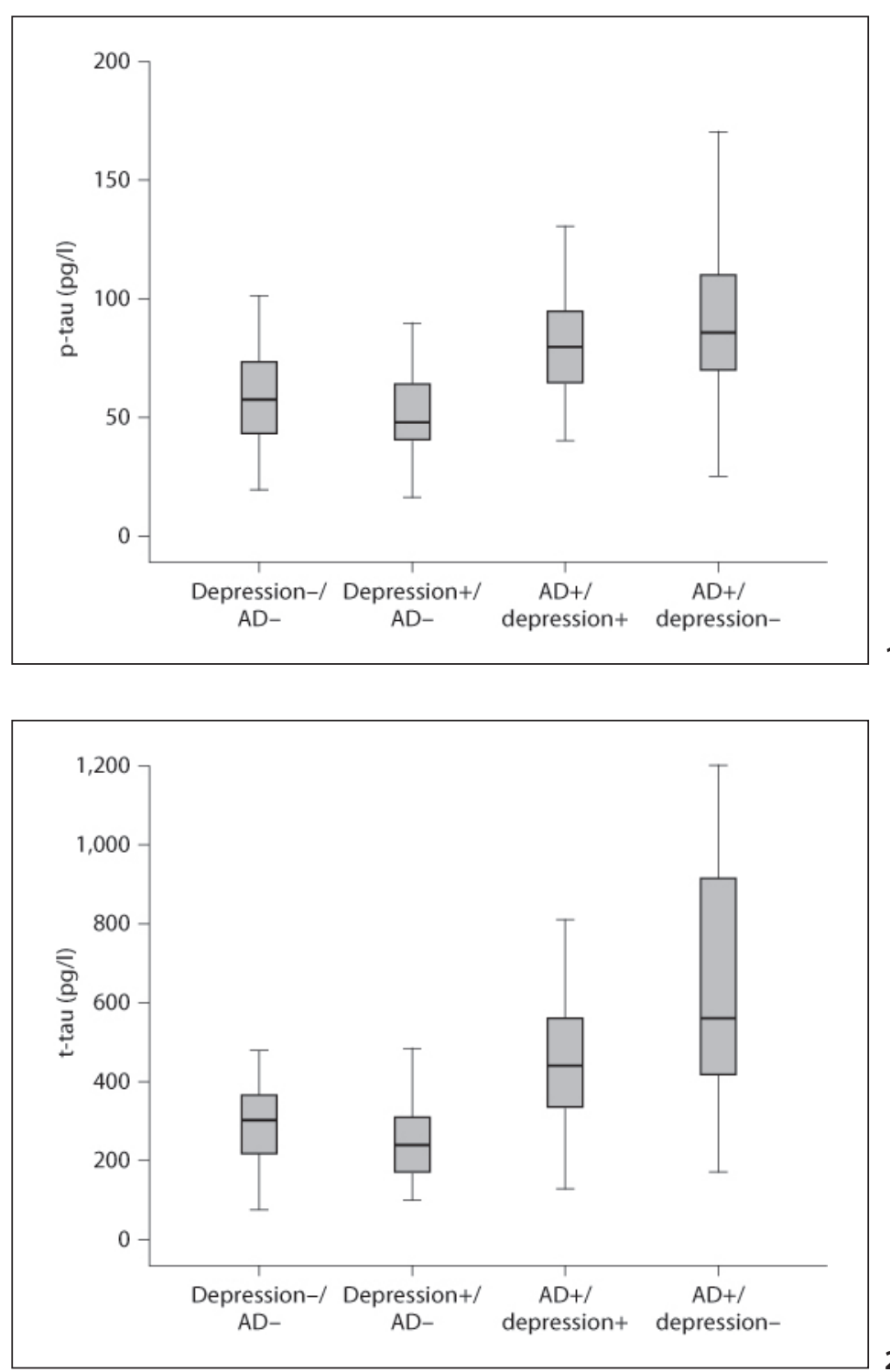

Fig. 1-3. Box plot of the three CSF markers in depressed and non-depressed patients with and without AD.

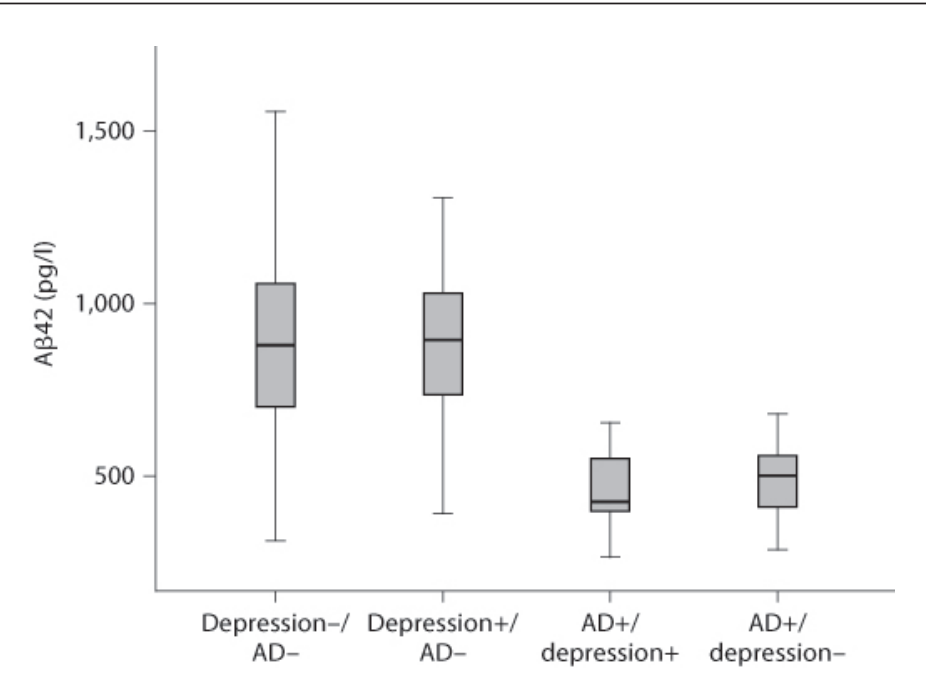


Table 3. Associations between CSDD scores and CSF measures

\begin{tabular}{|c|c|c|c|c|c|c|}
\hline \multirow[t]{2}{*}{ Independent variable: } & \multicolumn{2}{|c|}{$\mathrm{p}-\mathrm{tau} / 10$} & \multicolumn{2}{|c|}{$\mathrm{t}-\mathrm{tau} / 100$} & \multicolumn{2}{|c|}{$\mathrm{A} \beta 42 / 100$} \\
\hline & OR & $\mathrm{p}$ value & OR & $\mathrm{p}$ value & OR & $\mathrm{p}$ value \\
\hline Model 1 & 0.92 & 0.039 & 0.87 & 0.005 & 1.03 & 0.526 \\
\hline Model 2 & 0.92 & 0.071 & 0.85 & 0.008 & 0.98 & 0.811 \\
\hline
\end{tabular}

Ordered regression analysis. Model 1: adjusting for age and sex. Model 2: adjusting for age, sex, and MMSE score.

er than in the non-AD groups (all p values $<0.001$ ). T-tau differed significantly between AD patients with and without depression, with the depressed patients having lower values than the non-depressed AD patients, whereas p-tau and $A \beta 42$ did not differ significantly. In the SCI group, both t-tau and p-tau were significantly lower in the depressed compared to the non-depressed group, whereas $\mathrm{A} \beta 42$ did not differ significantly.

The correlation analyses were consistent with the above-mentioned findings. In the total group, bivariate correlations showed a significant negative correlation between the CSDD score and $\mathrm{p}$-tau (Spearman's $\rho=-0.21, \mathrm{p}=0.004)$ as well as $\mathrm{t}$-tau $(\rho=-0.25, \mathrm{p}=0.001)$, but not with $A \beta 42(p=0.25)$. In the AD group, there was a trend towards a correlation between the CSDD score and t-tau $(\rho=-0.21, p=0.05)$, whereas no significant correlations between the CSDD score and CSF measures were found in the SCI group. The ordered regression analyses, adjusting for age and sex (Model 1) and for age, sex, and MMSE score (Model 2), showed similar findings (table 3). Interpretations from Model 2 show that for each 10-pg/1 increase in $\mathrm{p}$-tau, the odds for having more depressive symptoms decreases by $8 \%$, and for each $100-\mathrm{pg} / \mathrm{l}$ increase in $\mathrm{t}$-tau, the odds for having more depressive symptoms decreases by $15 \%(\mathrm{p}=0.008)$.

\section{Discussion}

In this study, analysis of the three different CSF measures $A \beta 42$, $t$-tau, and $p$-tau did not support the hypothesis that more severe $\mathrm{AD}$ pathologies such as amyloid plaques or tau pathologies are associated with more severe depression. In contrast, depressed patients tended to have lower p-tau levels and, in particular, lower $\mathrm{t}$-tau levels than those without depression, indicating less tau pathology and neurodegeneration. These findings were found both in patients with a clinical diagnosis of AD and in subjects without dementia, using either continuous data or categories based on cut-off scores for the CSDD and reference norms for CSF values, and also after adjusting for potential confounders.

Few previous studies of CSF AD markers in patients with depression have been reported. One study reported on a population-based sample of 81 elderly women, 11 with major depression [16]. They were without dementia up to 10 years after lumbar puncture. A $\beta 42$ concentrations were higher among those with major depression compared to non-depressed women, whereas t-tau levels did not differ between the groups. p-tau was not measured in that study. In another study of patients with mild $\mathrm{AD}$, apathy was associated with higher ptau levels, but no associations between CSF A 342 , t-tau, or p-tau levels and the Neuropsychiatric Inventory depression item were found [15]. Thus, the current findings are consistent 
with previous CSF studies suggesting that AD pathology does not contribute to depression in elderly subjects with or without AD.

One previous clinicopathologic study reported more severe tau pathology in AD patients with comorbid depression compared to AD patients without depression [10]. Thus, it is possible that depression later in the disease course is more closely related to the underlying pathology, whereas other factors are more important in the earlier stages. However, the effect of depression on neurofibrillary tangle occurrence was rather small, and the interval between depression diagnosis at study inclusion and death was nearly 10 years [10]. In another autopsy study, 10 patients with late-onset major depression were followed prospectively to death [22]. AD was the most frequent pathology, in particular among the 7 patients who developed dementia, but Lewy body pathology and stroke were also common. Although the study shows that $\mathrm{AD}$ and other pathologies probably contribute to dementia in patients with late-onset major depression, whether these pathologies are related to the symptoms of depression was not reported. Finally, in a recent population-based clinicopathologic study of subjects who did not develop dementia during life, no association between AD-type changes and depression could be observed [13].

Amyloid imaging provides another means of exploring the association between depression and AD pathology. In one such study, patients with remitted depression underwent detailed neuropsychological assessment and Pittsburgh compound B (PiB) imaging [11]. Two subjects with normal cognition showed $\mathrm{PiB}$ retention in the normal range. In 3 of 6 patients with $\mathrm{MCI}$, the $\mathrm{PiB}$ retention fell in the range of $\mathrm{AD}$. However, associations between depression and $\mathrm{PiB}$ binding were not reported, and thus it is unclear whether the increased binding was related to cognitive impairment as a prodrome of $\mathrm{AD}$ or to the remitted depression [11]. In another PET study of non-demented subjects, a marker of amyloid plaques and neurofibrillary tangles was found to correlate with a self-report measure of depressive symptoms in both cognitively normal and MCI subjects [12].

Methodological limitations of the current study include the cross-sectional design; therefore, assumptions regarding causality cannot be made. The study sample was selected from referrals to a university memory clinic, and thus generalizations to the population are not possible. In particular, the non-AD group consisted of SCI subjects, with an increased proportion having an AD-type CSF profile [23], and hence extrapolation to non-demented depressed elderly persons is difficult. The diagnosis of AD was clinical, and thus there is a possibility of misdiagnosis. However, the lack of association between AD markers and depression was independent of $\mathrm{AD}$ diagnosis; therefore, inaccurate diagnosis is an unlikely explanation.

\section{Conclusion}

Our CSF analyses did not support the hypothesis that depression is associated with more $\mathrm{AD}$ pathology, neither in subjects with $\mathrm{AD}$ nor in subjects without $\mathrm{AD}$, suggesting that other factors are more important contributors to depression in the elderly. Future studies should explore the role of potential aetiological factors for depression in the elderly, such as neuroinflammation and cerebrovascular disease as well as psychosocial factors. 


\section{Disclosure Statement}

Dr. Aarsland serves on the scientific advisory board for DiaGenic; he has received honoraria from Lundbeck, Novartis, DiaGenic, GSK, and GE Health and research support from Novartis, Merck Serono, and Lundbeck.

\section{References}

1 Alexopoulos GS: Depression in the elderly. Lancet 2005;365:1961-1970.

-2 Byers AL, Yaffe K: Depression and risk of developing dementia. Nat Rev Neurol 2011;7:323-331.

-3 Caracciolo B, Backman L, Monastero R, Winblad B, Fratiglioni L: The symptom of low mood in the prodromal stage of mild cognitive impairment and dementia: a cohort study of a community dwelling elderly population. J Neurol Neurosurg Psychiatry 2011;82:788-793.

-4 Casanova MF, Starkstein SE, Jellinger KA: Clinicopathological correlates of behavioral and psychological symptoms of dementia. Acta Neuropathol 2011;122:117-135.

-5 Zubenko GS, Moossy J, Kopp U: Neurochemical correlates of major depression in primary dementia. Arch Neurol 1990;47:209-214.

-6 Nitsch RM, Wurtman RJ, Growdon JH: Regulation of APP processing. Potential for the therapeutical reduction of brain amyloid burden. Ann NY Acad Sci 1996;777:175-182.

-7 Nitsch RM, Deng M, Growdon JH, Wurtman RJ: Serotonin 5-HT2a and 5-HT2c receptors stimulate amyloid precursor protein ectodomain secretion. J Biol Chem 1996;271:4188-4194.

-8 Andreescu C, Butters MA, Begley A, Rajji T, Wu M, Meltzer CC, Reynolds CF 3rd, Aizenstein H: Gray matter changes in late life depression - a structural MRI analysis. Neuropsychopharmacology 2008;33:2566-2572.

-9 Rapp MA, Schnaider-Beeri M, Grossman HT, Sano M, Perl DP, Purohit DP, Gorman JM, Haroutunian V: Increased hippocampal plaques and tangles in patients with Alzheimer disease with a lifetime history of major depression. Arch Gen Psychiatry 2006;63:161-167.

-10 Rapp MA, Schnaider-Beeri M, Purohit DP, Perl DP, Haroutunian V, Sano M: Increased neurofibrillary tangles in patients with Alzheimer disease with comorbid depression. Am J Geriatr Psychiatry 2008;16:168-174.

-11 Butters MA, Klunk WE, Mathis CA, Price JC, Ziolko SK, Hoge JA, Tsopelas ND, Lopresti BJ, Reynolds CF 3rd, DeKosky ST, et al: Imaging Alzheimer pathology in late-life depression with PET and Pittsburgh Compound-B. Alzheimer Dis Assoc Disord 2008;22:261-268.

-12 Lavretsky H, Siddarth P, Kepe V, Ercoli LM, Miller KJ, Burggren AC, Bookheimer SY, Huang SC, Barrio JR, Small GW: Depression and anxiety symptoms are associated with cerebral FDDNP-PET binding in middle-aged and older nondemented adults. Am J Geriatr Psychiatry 2009;17:493-502.

-13 Tsopelas C, Stewart R, Savva GM, Brayne C, Ince P, Thomas A, Matthews FE: Neuropathological correlates of late-life depression in older people. Br J Psychiatry 2011;198:109-114.

-14 Mattsson N, Zetterberg H, Hansson O, Andreasen N, Parnetti L, Jonsson M, Herukka SK, van der Flier WM, Blankenstein MA, Ewers M, et al: CSF biomarkers and incipient Alzheimer disease in patients with mild cognitive impairment. JAMA 2009;302:385-393.

-15 Skogseth R, Mulugeta E, Jones E, Ballard C, Rongve A, Nore S, Alves G, Aarsland D: Neuropsychiatric correlates of cerebrospinal fluid biomarkers in Alzheimer's disease. Dement Geriatr Cogn Disord 2008;25:559-563.

-16 Gudmundsson P, Skoog I, Waern M, Blennow K, Palsson S, Rosengren L, Gustafson D: The relationship between cerebrospinal fluid biomarkers and depression in elderly women. Am J Geriatr Psychiatry 2007;15:832-838.

-17 Alexopoulos GS, Abrams RC, Young RC, Shamoian CA: Cornell Scale for Depression in Dementia. Biol Psychiatry 1988;23:271-284.

-18 Alexopoulos GS, Abrams RC, Young RC, Shamoian CA: Use of the Cornell scale in nondemented patients. J Am Geriatr Soc 1988;36:230-236.

-19 Korner A, Lauritzen L, Abelskov K, Gulmann N, Marie Brodersen A, Wedervang-Jensen T, Marie Kjeldgaard K: The Geriatric Depression Scale and the Cornell Scale for Depression in Dementia. A validity study. Nord J Psychiatry 2006;60:360-364. 
-20 Jorm AF, Jacomb PA: The Informant Questionnaire on Cognitive Decline in the Elderly (IQCODE): socio-demographic correlates, reliability, validity and some norms. Psychol Med 1989;19:1015-1022.

-21 Reisberg B, Ferris SH, de Leon MJ, Crook T: The Global Deterioration Scale for assessment of primary degenerative dementia. Am J Psychiatry 1982;139:1136-1139.

-22 Sweet RA, Hamilton RL, Butters MA, Mulsant BH, Pollock BG, Lewis DA, Lopez OL, DeKosky ST, Reynolds CF 3rd: Neuropathologic correlates of late-onset major depression. Neuropsychopharmacology 2004;29:2242-2250.

-23 Visser PJ, Verhey F, Knol DL, Scheltens P, Wahlund LO, Freund-Levi Y, Tsolaki M, Minthon L, Wallin AK, Hampel H, et al: Prevalence and prognostic value of CSF markers of Alzheimer's disease pathology in patients with subjective cognitive impairment or mild cognitive impairment in the DESCRIPA study: a prospective cohort study. Lancet Neurol 2009;8:619-627. 